\title{
MODELO COGNITIVO COMPORTAMENTAL DEL SÍNDROME DE NARCOLEPSIA-CATAPLEJÍA: EXPOSICIÓN TEÓRICA
}

\author{
H. ANDRÉS MARÍN ${ }^{1.2}$ Y STEFANO VINACCIA ${ }^{1}$ \\ ${ }^{1}$ Grupo de Salud Comportamental, Universidad de San Buenaventura, Medellín, Colombia \\ ${ }^{2}$ Unidad de sueño, Grupo de Neurociencias, Universidad de Antioquia, Colombia
}

(Aceptado en febrero de 2005)

\begin{abstract}
El reciente desarrollo de la medicina comportamental del sueño, ha llevado a diferentes autores a pensar en la conceptuación explicativa de las diferentes patologías del sueño. Si bien es cierto que se ha avanzado en los diferentes modelos explicativos del sueño, como el modelo interactivo conductual, el modelo comportamental del sueño, el modelo de regulación del sueño, también es cierto que las conceptualizaciones comportamentales, hasta ahora y por lo general, habian estado de lado de comprensión del fenómeno del insomnio, y las tendencias explicativas de la somnolencia excesiva diurna, son de aparición reciente. El objetivo de este estudio, es hacer una revisión teórica del planteamiento cognitivo comportamental del síndrome de nercolepsia cataplejía, planteado recientemente, analizando los puntos centrales de dicho modelo, en la explicación y la intervención de la narcolepsia, como aporte importante dentro de la medicina comportamental del sueño.
\end{abstract}

Palabras clave: Narcolepsia, cataplejía, parálisis del sueño, alucinaciones hipnagógicas, modelo comportamental del sueño, medicina comportamental del sueño.

\section{Cognitive-behavioml model of narcolepsy-cataplexy syndrome: $A$ theoretical view}

Recent developments in behavioral sleep medicine have been made concerning conceptualization and explanation of different sleep disorders. Although it is certain that advances have been made in relation to different explanatory sleep models, such as the behavioral interactive model, the behavioral sleep model, and the sleep regulation model, it is also true that behavioral conceptualizations suggested until now had been focused on the comprehension of the phenomenon of insomnia. However, explanatory models of day time sleepiness have emerged only recently. In this study we review the cognitive-behavioral formulation of the narcolepsy-cataplexy syndrome. A model is presented, analyzing the central points of such model in relation to explanation and intervention aspects of narcolepsy, and suggesting the model as an important contribution to behavioral sleep medicine.

Key words: Narcolepsy, cataplexy, sleep paralysis, hipnagogic hallucinations, behavioral sleep model, behavioral sleep medicine.

\section{INTRODUCCIÓN}

El síndrome de narcolepsia cataplejía es quizá el único trastorno del sueño, con un componente biológico intrínseco, que altera esta función (Dement y Vaughan, 2000). Es posible y quizá sea esta la razón principal por la cual hasta ahora, no se había

Correspondencia: Hernán Andrés Marín, Grupo de Salud Comportamental, Universidad de San Buenaventura, Medellín, Colombia. Correo-e: behavioralsleepmedicine@yahoo.com.ar pensado en una explicación psicológica del mismo. Desde los diferentes enfoques psicológicos, se había pensado el síndrome de narcolepsia, desde un plano investigativo, en las consecuencias psicosociales del síndrome (Dinges, 1989; Ramos, 1998, Marín y Vinaccia, 2004b), debido a que es una enfermedad crónica y las consecuencias tanto para el paciente como para la familia, son difíciles de afrontar. Otros estudios, han indagado la probabilidad de accidentes laborales y de transito 
en los pacientes narcolépticos; Las funciones neuropsicológicas, afectadas por este trastorno del sueño, en cuanto al tiempo de reacción, funciones ejecutivas y memoria; la calidad de vida relacionada con la salud, entre otras (Dinges, 1989; Conesa et al., 1998; Bladin, 2000; Daniels et al. 2001; Dement y Vaughan, 2000; Ramos, 1998; Marín y Vinaccia, 2004a; Cano Miró et al., 2004). Sin embargo, la conceptualización psicológica del síndrome (sin el ánimo de psicologizarlo), no se había abordado hasta ahora. Esto es curioso, puesto que si bien es cierto las características patogénicas biofisiológicas del síndrome, son claras, las manifestaciones conductuales, factores emocionales que detonan la cataplejía, hacen pensar en la influencia de los factores ambientales en las características intrínsecas del síndrome, como la respuesta ante situaciones emocionales intensas de las manifestaciones catapléjicas, lo mismo que los ataques de sueño, ligados a situaciones de arousal alto; las cuales pueden tener una explicación cognitivo conductual, apoyada también en las explicaciones que desde este modelo teórico se han hecho del sueño, y en las explicaciones de la dinámica y regulación del mismo que han hecho deferentes autores especializados en el tema (Rogers y Mullington, 2003; Marín, 2001b; Hublin et al. 1994; Mamelak, 1992; Okun et al. 2002; Scammell, 2003). Para el abordaje de este aspecto es necesario describir los diferentes modelos teóricos que han apuntado, tanto desde lo fisiológico, como lo orgánico, a describir el síndrome; lo mismo que definir aquellos modelos psicológicos que han explicado al sueño, y la somnolencia excesiva diurna.

\section{UNA VISIÓN HISTÓRICA DE LA NARCOLEPSIA}

La narcolepsia no ha sido ajena al desarrollo histórico y teórico en la explicación que se ha dado a los diferentes aspectos relacionados con la salud y la enfermedad. Desde la denominación del síndrome a partir de Gelineau (1880), sus predecesores Willis, Graves, Westphalt, entre otros, han jugado un papel importante en la descripción del síndrome narcolepsiacataplejía. En sus inicios, Galineau, denominó narcolepsia a «Una neurosis rara o poco conocida....caracterizada por una necesidad imperiosa de dormir, súbita y de corta duración, que se reproduce en intervalos más o menos largos» (Gelineau, 1880), esta afirmación es posible identificarla con las manifestaciones sintomáticas principales de la narcolepsia, como lo es la cataplejía y la somnolencia excesiva diurna. Las primeras explicaciones de la narcolepsia, no diferenciaban este síndrome de la neurosis o de la encefalitis. Sólo hasta 1926, Adie definiría la narcolepsia idiopática, como una entidad específica, sentando así las bases para diferenciarla de la epilepsia y de los otros síndromes anteriores, pero con pocas bases neuroanatómicas y neurofisiológicas, situación comprensible debido a que hasta ese momento, no se había hecho efectivos los desarrollos tecnológicos necesarios dentro del surgimiento de la medicina del sueño (Gelineau, 1880; Yoss y Daly 1960; Loewenfeldt, 1902; Kripke, 1976; Mignot, 2000). Mientras tanto solo se poseían las explicaciones de Gelineau a cerca de la perdida del tono muscular (cataplejía, la cual denominaría atasia), y el termino de cataplejía a parecería definido más adelante con Löwenfeldt. Otro avance significativo aparecería posteriormente (teniendo en cuenta las explicaciones previas), a través de Yoss y Dalí, quienes definirían sintomáticamente la tétrada narcoléptica, formada por los ataques de sueño, la cataplejía, la parálisis de sueño y la intrusión del sueño REM (de la sigla inglesa Rapid Eye Movement, movimientos oculares rápidos, conocido también como sueño paradójico) o las 
ensoñaciones en la vigilia, denominadas, alucinaciones hipnagógicas; convirtiéndose en los primeros criterios diagnósticos, para la descripción sintomática de la narcolepsia (Gelineau 0, 1880; Yoss y Daly 1960; Loewenfeldt, 1902; Kripke, 1976; Mignot, 2000).

En 1975 se organiza se organiza el primer simposio internacional de narcolepsia en Francia y se queda de acuerdo en la definición del síndrome, como «un síndrome del cual no se conoce el origen y caracterizado por una tendencia anormal al sueño, incluyendo somnolencia excesiva diurna....manifestaciones patológicas del sueño REM y puede incluir una disociación, entre los procesos inhibitorios del sueño REM, cataplejía, parálisis de sueño, excesiva somnolencia diurna y alucinaciones hipnagógicas. Esta definición ha estado presente en la descripción del síndrome a través de los diferentes sistemas de Clasificación internacional de las patologías del sueño (en adelante, ICDS) de la Asociación Americana de Medicina del Sueño (AAMS) en sus revisiones (1979, 1990, 1997, 2001 y 2005) y conserva gran parte de estas hipótesis, claro esta que las revisiones, hacen referencia a aspectos centrales, en el desarrollo de la compresión de la narcolepsia, como son los factores epidemiológicos (los cuales aluden al $0,05 \%$ de la población), factores genéticos e inmunitarios (asociación al antígeno HLA-DR2), bases anatomo-fisiológicas (donde se involucra el núcleo pontino, entre otras (Gelineau, 1880; Yoss y Daly 1960; Loewenfeldt, 1902; Kripke, 1976; Mignot, 2000).

\section{DESCRIPCIÓN SINTOMÁTICA DEL SÍNDROME}

De acuerdo a la historia de la conceptualización del síndrome y al planteamiento de clasificaciones internacionales de las patologías mentales y del sueño, los siste- mas Manual diagnóstico y estadístico de los trastornos mentales DSM-IV-TR, y la Clasificación internacional de las patologías del sueño, ICDS II 2005, plantean una descripción central del síndrome, basada en la famosa tétrada narcoléptica: somnolencia excesiva diurna, cataplejía, parálisis de sueño y alucinaciones hipnagógicas (Estas dos últimas, consideradas como intrusiones de sueño REM) (American Academy of Sleep Medicine 2005, American Psychiatry Association, 2002). El primer síntoma, somnolencia excesiva diurna, es definida como crisis de sueño y episodios de sueño de corta duración (micro sueño), este es el síntoma principal y en la mayoría de los casos el primero en manifestarse en el curso del trastorno. Los ataques de sueño se manifiestan en forma repentina y son de breve duración (de 2 a 5 minutos), y desaparecen con la misma rapidez, el afectado se despierta fresco y con perdida de memoria anterógrada, de lo ocurrido, durante el episodio de sueño. El segundo síntoma de la tétrada narcoléptica, es la cataplejía, se entiende por este termino una repentina laxitud de la musculatura, que ocurre en el estado de vigilia, en algunos pacientes solo afecta determinado grupo muscular y en otros pacientes toda la actividad músculo esquelética, normalmente dura unos cuantos segundos o minutos, pero puede llegar $s$ a su duración total de 30 minutos, descritos en algunos casos (American Academy of Sleep Medicine 2005, American Psychiatry Association, 2002). Corrientemente, los factores desencadenantes, son emociones o excitación, como, por ejemplo, alegría y risa, aunque también por enfados, especialmente cuando las sensaciones se manifiestan repentinamente y por sorpresa. La parálisis de sueño, otro de los síntomas, hace su aparición inmediatamente antes de quedarse dormido el sujeto, durante la vigilia nocturna, o tras despertarse en la mañana. En este caso también se trata de una incapacidad 
repentina y pasajera para moverse, pero al contrario de la cataplejía, su desencadenamiento no se debe a emociones. El ultimo síntoma central de la narcolepsia, son las alucinaciones hipnagógicas, que se tratan de activas alucinaciones antes del comienzo del sueño, que pueden causar un gran sobresalto (American Academy of Sleep Medicine 2005; American Psychiatric Association, 2002).
Existen otros síntomas secundarios a la narcolepsia, como la automatización de conductas, especialmente cuando el sujeto, a pesar de experimentar la sensación de somnolencia, intenta continuar con su actividad momentánea. Hallazgos polisomnográficos, detectan una latencia corta de sueño con arquitectura REM de inicio o una latencia breve de esta, entre otros síntomas. La Tabla 1 describe los criterios

Tabla 1. Clasificaciones diagnósticas de la narcolepsia, de acuerdo a la clasificación internacional de enfermedades mentales DSM-IV-TR (2002) y la clasificación internacional de trastornos del sueño ICSD-II (2005)

\begin{tabular}{|c|c|}
\hline & Criterios diagnósticos para el \\
\hline & DSM-IV-TR \\
\hline A. & $\begin{array}{l}\text { Ataques de sueño reparador irresistibles que } \\
\text { aparecen diariamente durante un mínimo de } \\
\text { tres meses. }\end{array}$ \\
\hline B. & $\begin{array}{l}\text { Presencia de uno do ambos de los siguientes } \\
\text { síntomas: }\end{array}$ \\
\hline & $\begin{array}{l}\text { (1) Cataplejía (es decir episodios breves y } \\
\text { súbitos de perdida bilateral del tono } \\
\text { muscular, la mayoría de veces en } \\
\text { asociación con emociones intensas). } \\
\text { (2) Intrusiones recurrentes de elementos del } \\
\text { sueño REM, en las fases de transición } \\
\text { entre el sueño y la vigilia, tal y como } \\
\text { indican alucinaciones hipnagógicas o } \\
\text { hipnopompicas, o las parálisis del sueño } \\
\text { al principio o al final de los episodios del } \\
\text { sueño. }\end{array}$ \\
\hline
\end{tabular}

C. La alteración no se debe a los efectos fisiológicos directos de una sustancia p.ej., Drogas o Fármacos), o de una enfermedad médica
A. El paciente tiene una queja de somnolencia excesiva diurna o debilidad muscular repentina
B. Siestas o ataques recurrentes de sueño casi diariamente por lo menos 3 meses.
C. La pérdida bilateral repentina de tono postural del músculo que ocurre en asociación con una emoción intensa (cataplejía).
D. Características asociadas que incluyen:
1. La parálisis del sueño
2. Alucinaciones hipnagógicas
3. Comportamientos automáticos
4. Disrupción del episodio del sueño mayor

E. Hallazgos polisomnográficos, que demuestran uno o más de los siguientes síntomas.

1. Latencia de sueño menos de 10 minutos

2. Latencia de sueño REM menos de $\mathbf{2 0}$ minutos

3. En el Test de Múltiple Latencia de Sueño (TMLS), que demuestre una latencia de sueño, estable de menos de 5 minutos.

4. Dos o más registros de periodos de sueño REM, al inicio del sueño, durante el estudio TMLS

F. Tipificación HLA que demuestre la positividad DQB1*0602 o DR2.

G. Ningún desorden médico o mental explica los sintomas.

H. Otro trastorno del sueño (p.ej., trastorno del movimiento períbdico de las extremidades o síndrome central del apnea del sueño) puede estar presente, pero no es la causa primaria de los síntomas.

HLA DQB1 * 0602 o DR2 (complejo mayor de histocompatibilidad leucocitario, de la clase designada DQ, DR). En la tabla 1, se evidencia que se conserva el acuerdo de la sintomatología de la narcolepsia en la tétrada, Somnolencia excesiva diurna, cataplejía, parálisis de sueño y alucinaciones hipnagógicas, síntomes en los cuales se centrarán el planteamiento del modelo comportamental. la diferencia más visible en las dos clasificaciones es la inclusión de los parámetros polisomnográficos y evaluación genética, en la descripción del síndrome de la ICDS. 
diagnósticos de los sistemas de clasificación más comunes, para la narcolepsia, en donde se evidencia claramente el acuerdo en la primera tétrada de síntomas antes descrita, y se amplían otros síntomas de origen polisomnográfico, los cuales quizás sean la diferencia central entre los dos sistemas de clasificación.

\section{HACIA UNA}

COMPRENSIÓN DE UN MODELO COGNITIVO-COMPORTAMENTAL DEL SÍNDROME NARCOLEPSIA CATAPLEJÍA

Sin lugar a dudas el desarrollo explicativo de la psicología con respecto a los trastornos del sueño, ha estado más de la mano de las teorías explicativa a cerca del insomnio y los ritmos circadianos, en donde las diferentes revisiones teóricas interventivas y explicativas, apuntan a una mayor producción en este aspecto (Marín, 2001b). Recientemente el desarrollo de la medicina comportamental del sueño y de las explicaciones de los modelos psicológicos del mismo, han propiciado un acercamiento tanto de intervención, como explicativo de otros trastornos del sueño diferentes al insomnio, y es así como se proponen teorías explicativas e interventivas de la somnolencia excesiva diurna, como síndrome con causas fisiológicas, moduladas por aspectos exógenos (Rogers y Mullington, 2003; Marín, 2001b). Esto permite analizar y proponer un modelo del síndrome de narcolepsia cataplejía, pensando en estas características y afirmando las razones interventivas, que en la bibliografía de la intervención del mismo, reconocen los expertos como parámetros importantes dentro de la intervención de la narcolepsia desde el plano psicológico. Para hacer un análisis explicativo de la narcolepsia dentro de la concepción cognitivacomportamental, es importante, retomar algunos elementos que aportan los modelos que hasta ahora han explicado el sueño y en especial la narcolepsia como son, el modelo neuroanatómico de la narcolepsia de Stanford, el modelo objetivo comportamental del sueño, el modelo interactivo comportamental del sueño y el modelo interactivo comportamental de la somnolencia excesiva diurna.

Durante el planteamiento del modelo neuroanatómico de la narcolepsia (Aldrich et al., 1991,1992, 1993, 1994; Boehme et al., 1984; Carlander et al., 1993; Carpizo et al., 1998; Dement, et al. 1966; Mignot, 2000, 2001; Mignot et al., 1988,1989,1993,1998; Lock et al.,1998), las teorías en principio, consideraban al síndrome, como una enfermedad exclusiva del sueño REM (Mignot, 2000, 2001); posteriormente fue definida como una alteración del ritmo vigilia-sueño, hipótesis que se vendría abajo, al describir que los ritmos circadianos de la temperatura, cortisol o la melatonina y los ritmos ultradianos en pacientes narcolépticos, se preservan (Carpizo et al., 1998). Más adelante se relacionaría la narcolepsia con un defecto en el control de los límites del estado de vigilancia, y recurrentemente se han propuesto dos nuevas hipótesis, la de disregulación homeostásica, y la teoría autominmune (Carpizo et al., 1998). Las investigaciones direccionadas a corroborar la primera hipótesis, mediante el análisis de la densidad espectral de la actividad lenta, realizados en condiciones basales y tras la deprivación de sueño, han demostrado que los pacientes narcolépticos son más sensibles a las condiciones ambientales pero no se observan alteraciones homeostásicas del sueño NREM (de la sigla inglesa, no rapid eyes movements, en español sueño de ondas lenta o sueño donde no existen movimientos oculares rápidos). Por la misma dirección (Mignot et al., 1988, 1989, 1993, 1998), la narcolepsia parece no ser una enfermedad autoinmune clásica, si bien los estu- 
dios sobre la población celular microgrial y las citocinas que producen el sueño, parecen arrojar resultados alentadores, de todas formas el tema de la fisiopatología de la narcolepsia, sigue siendo un tema de debate, donde todos los datos apuntan a la descripción de una alteración de los mecanismos neuroquímicos reguladores del sueño (Lock et al.,1998). Desde este punto el modelo neuroquímico de la narcolepsia, surge en Stanford, partiendo del modelo explicativo sintomático de la narcolepsia canina, para posteriormente ubicarlo en explicaciones neuroanatómicas del mismo (Mignot, 2000, 2001). Diferen- tes investigaciones en animales, han demostrado que la interacción de la hipocretina y su déficit producen Narcolepsia. En éste modelo, el control neuroquímico, y neurofarmacológico de la narcolepsia, se explica por las alteraciones genéticas del sistema hipocretínico, las cuales por la hipoactividad dopaminérgica, o hiperactividad colinérgica, producen una manifestación sintomática del síndrome (ver Figura 1).

La hipótesis encontrada en los modelos animales de la narcolepsia, a cerca de la patogénesis del síndrome, debido a deficit de hipocretina, radica en la

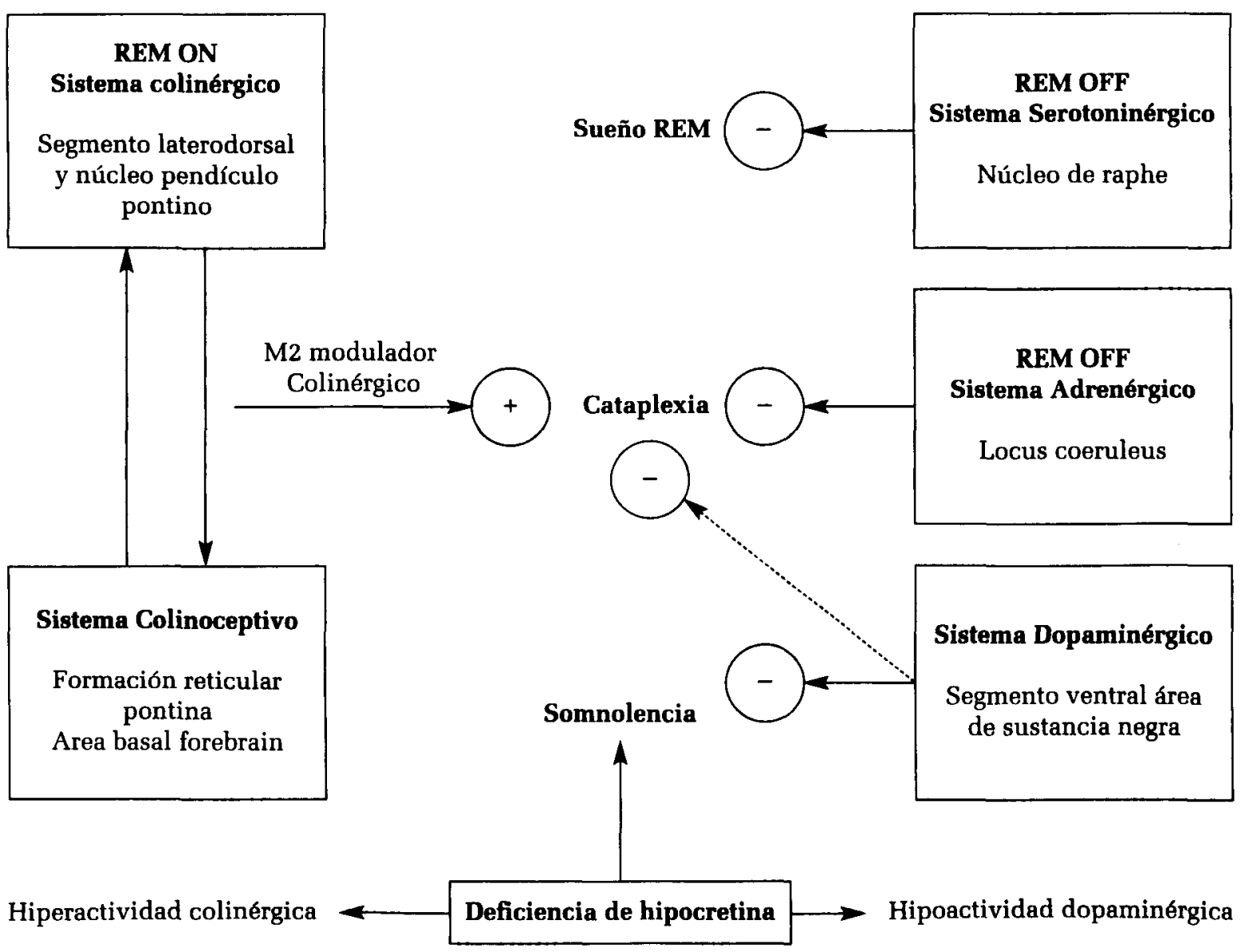

Figura 1. Modelo neuroanatómico y neuroquímico de la narcolepsia (Mignot, 2000), donde se observa como la defíciencia hipocretínica incide en las manifestaciones sintomáticas neuroquímicas del sindrome, aumentando o disminuyendo, algunos de sus componentes. REM ON (activación del sueño REM), REM OFF (inhibición del sueño REM), M2 (modulador del sistema colinérgico). 
pregunta de cómo los péptidos hipotalámicos, interactúan con los grupos neuronales monoaminérgicos y colinérgicos, generando somnolencia excesiva diurna y sueño REM anormal. Los neurotransmisores de hipocretina, se proyectan más fuertemente sobre el locus coeruleus, excitado de forma natural. Por lo general esta estructura inhibe la producción de sueño REM, y al decrementar la actividad del locus coeruleus, por el decremento de la hipocretina, desinhibe el sueño REM y produce la narcolepsia.

El modelo de regulación de sueño (Achermann y Borbely, 2003), o modelo de triple procesador, es una propuesta de modelo biológico, el cual sostiene que el sueño está regulado por tres procesos, que dan cuenta de la desincronización fraccional que puede darse entre los ciclos de actividad -descanso, sueño- vigilia. El proceso $S$, se refiere a la necesidad de sueño que aumenta en la medida que la persona está despierta, necesidad que no es absoluta y se modula con preferencias, horas y demandas sociales y que daría cuenta durante el día, del déficit de sueño de sueño acumulado. El proceso C, Explica la alternancia regular de periodos de sueñovigilia, regulados por sincronizadores endógenos rítmicos. Por último el proceso $W$, re refiere a la inercia de sueño, que se manifiesta en los primeros minutos después del despertar y que una vez finalizado el sueño, persiste por cierto tiempo (Figura 2).

El modelo comportamental del sueño (Webb, 1988), sostiene que existen tres sistemas de variables primarias, que intervienen en la respuesta del sueño y son la demanda del sueño, los factores comportamentales que facilitan o inhiben el sueño y los ritmos biológicos. Estas variables primarias son a la vez función de varias variables secundarias, como son la edad, las diferencias de la

\section{TENDENCIA DEL SUEÑO}
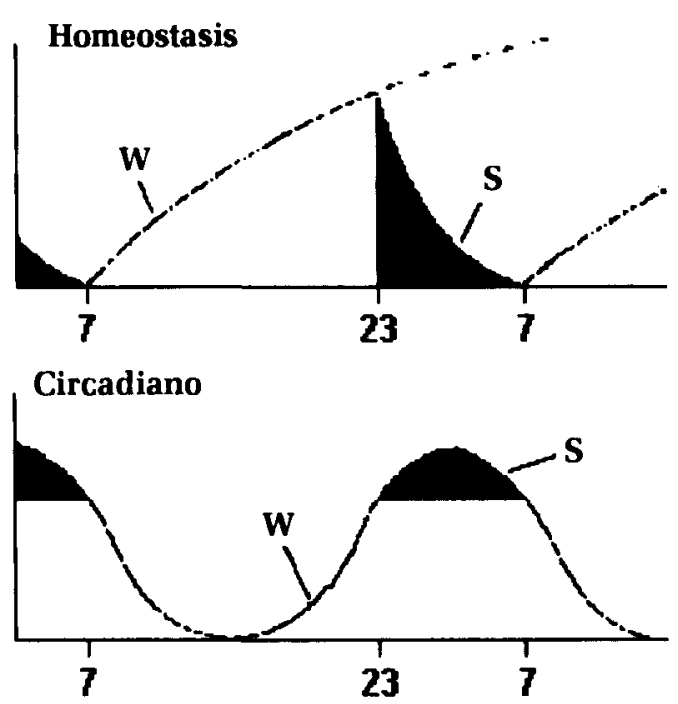

Ultradiano

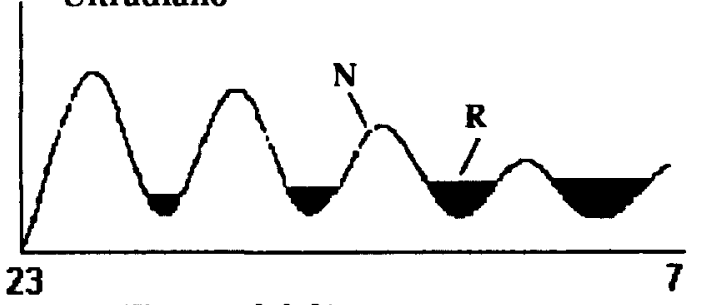

Tiempo del día

Figura 2. Modelo de regulación del sueño, que ilustra los tres procesos centrales en la regulación del sueño (Bórbely, 2000) En la Figura se percibe claramente la periodicidad, de cada uno de los procesos de regulación de sueño: $S$ (necesidad de sueño), W (inercia de sueño). En el proceso ultradiano, se observa el decremento tradicional del sueño NREM $(\mathrm{N})$ y el aumento del sueño $R E M(R)$, situación que ocurre periódicamente a lo largo de la noche en la arquitectura del sueño normal.

especie, así como de diferencias individuales en horarios y cronotipos y variables de personalidad, que modulan las variables primarias. De la modulación de ambas se producen los patrones de sueño, arquitectura y respuesta valorativa el mismo. En la siguiente tabla se observa la interacción de las variables antes mencionadas, y las subvariables que se agrupan en cada una de ellas explicando la 
operacionalización del proceso central (Tabla 2).

El modelo interactivo conductual (Buela-Casal y Sierra, 1995), retomando algunos conceptos de Webb, concibe que el tiempo total de sueño y la estructura del mismo, están determinados por la interacción de cuatro factores (a diferencia de la concepción exponencial de Webb): el tiempo circadiano (cuándo duerme), el organismo (cómo duerme), el ambiente (dónde duerme) y la conducta (qué hace para dormir). Este modelo fue descrito inicialmente como formulación para el insomnio. Pero ha demostrado que es oportuno al momento de evaluar otras patologías del sueño. en la siguiente Figura 0, se exponen claramente los factores descritos y en las líneas se aprecia la interacción de dichos procesos (Figura 3).

La somnolencia excesiva diurna, es otro

Tabla 2. Modelo comportamental del sueño, donde se observa la interacción de las variables que estructuran la respuesta del sueño (Webb, 1988). Se indica en primer lugar la manifestación objetiva y matemática de la respuesta del sueño, en donde el autor se refiere a una manifestación exponencial del mismo, de acuerdo a un modelo de regresión validado. En segundo lugar se explican las variables implicadas en la formula propuesta.

MODELO COMPORTAMENTAL DEL SUEÑO

$\mathbf{R}$ sueño $=$ demanda de sueño $\times$ tendencia circadiana \pm facilitadores $\mathrm{e}$ inhibidores conductuales

Respuesta de sueño = latencia de sueño, tiempo de despertar, estructura del sueño (estados y continuidad), y respuestas subjetivas

Demanda de sueño = Tiempo de vigilia (positivo) y tiempo de sueño (negativo)

Tendencia circadiana $=$ Tiempo circadiano del sueño

Facilitadores e inhibidores conductuales $=$ Voluntarios e involuntarios, compatibles e incompatibles con las respuestas del sueño.

Las variables primarias están moduladas por: diferencias en las especies, estado de desarrollo mental, estado organísmico y diferencias individuales

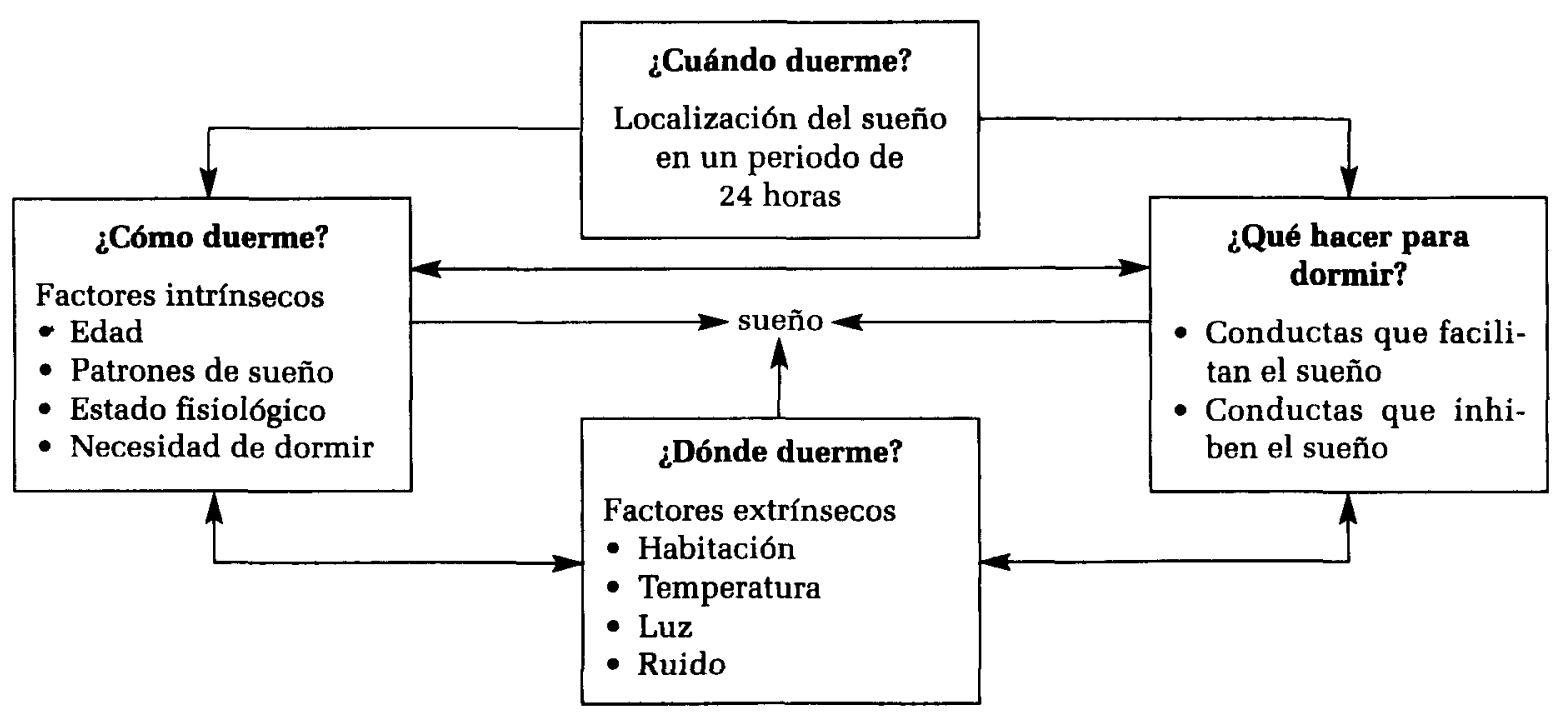

Figura 3. Modelo interactivo conductual del sueño (Buela-Casal y Sierra 1995). Las flechas conectan claramente los factores implicados en la producción del sueño, produciendo una interacción en los mismos, interacción que hace posible la manifestación de la conducta del sueño. 
de los síntomas más comunes de la narcolepsia y otros trastornos del sueño, por los cuales los pacientes acuden a terapia. La aparición de los modelos neuroconductuales de regulación de sueño (antes explicado, ver modelo de Borbely, 2000), y la influencia de sus componentes en la producción del mismo, llevó a diferentes autores a pensar en la forma de cómo se produce la somnolencia excesiva (Akerstedt y Folkard 1990; Folkard, Akerstedt, 1992; Dinges y Kribbs, 1991), y la función de la fragmentación del sueño en la producción del déficit de sueño acumulado y su relación con la somnolencia excesiva (Stepanski, 2002), además de los aspectos circadianos de la misma (Dinges, 1989; Monk, 1991); considerando aspectos, que pueden predisponer, precipitar y mantener el complejo fenómeno de la somnolencia excesiva diurna. Retomando estos aspectos el modelo comportamental de la somnolencia excesiva diurna (Marín, 2001b; Marín y Vinaccia 2004a), concibe la somnolencia excesiva como una respuesta subjetiva caracterizada por un deseo irresistible de dormir, en circunstancias inapropiadas o no deseadas, determinada por tres variables conductuales en la estructuración de la misma: (1) Variables situacionales que alteran o fragmentan el sueño nocturno, estas variables están determinadas por aquellas conductas que causan privación de sueño y que van moduladas en consonancia con el modelo de regulación de sueño y factores circadianos del mismo. (2) creencias o pensamientos que fragmentan el sueño, estos pensamientos están basados prácticamente en cogniciones y evaluaciones negativas sobre la eficacia y necesidad de sueño, además de cogniciones que favorecen la conducta activista y el estado de alerta más allá de los patrones circadianos (Pilcher, 2000; Marín, 2005). (3) características fisiológicas que fragmentan el sueño nocturno, algunos de estas son patogénesis de tipos específicos de hipersomnias (déficit de hipocretina en la narcolepsia, características morfofisiológicas en la apnea obstructiva de sueño, entre otras), otras referidas a la arquitectura de sueño y ciertas variables relacionadas con la calidad de sueño como: latencia de sueño, considerada como la medida de sueño en

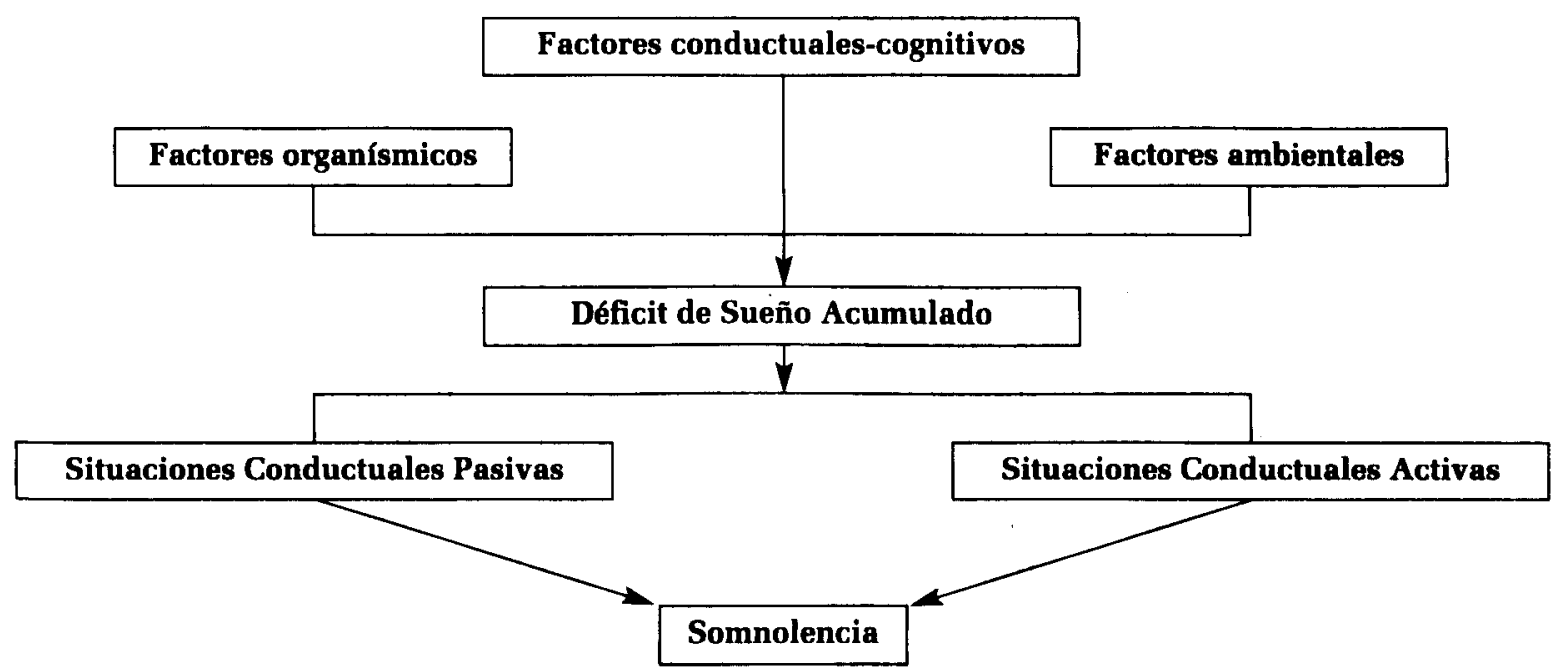

Figura 4. Modelo cognitivo comportamental interactivo de la somnolencia excesiva diurna, modificado (Marín, 2001b; Marín y Vinaccia, 2004b). Se aprecia, la interacción de factores centrales, orgánicos, conductuales-cognitivos y ambientales, en la producción del déficit de sueño acumulado y posteriormente antela presencia de situaciones conductuales activas o pasivas, la manifestación de la conducta de somnolencia diurna 
la cual una persona tarda en dormirse; duración de sueño, que hace referencia a la cantidad de horas que el sujeto es capaz de mantenerse dormido; eficiencia habitual de sueño, que hace referencia a la valoración de porcentaje de sueño que un sujeto quiere estar dormido, sobre el total de tiempo que permanece acostado, multiplicado por cien (Marín, 2001b; Marín y Vinaccia, 2004a). Estas variables modulan en interacción, con otras variables que fragmentan el sueño como una tendencia más fuerte a desarrollar somnolencia excesiva diurna. Los factores antes mencionados, apuntan a describir en su déficit, aspectos centrales que en general producen privación de sueño y como resultado la manifestación conductual del sueño excesivo, ante situaciones pasivas (aquellas que exigen un nivel de arousal y motivación bajo) y activas (las que exigen un nivel de arousal alto), con una caracterización de acuerdo a su curso (de acuerdo a la clasificación internacional de trastornos del sueño ICSD-2), de significación clínica, leve, moderada y grave; y sus consecuencias, como se observa en la grafica siguiente (Marín, 2001b; Marín y Vinaccia 2004a).

Un aporte complementario al modelo de la somnolencia excesiva diurna, es el microanálisis comportamental de la manifestación sintomática de la misma, que a diferencia del modelo conductual interactivo, que explica el surgimiento o etiología de la somnolencia, refiriéndose a conductas, que pueden exacerbar los síntomas orgánicos, o manifestar el comportamiento sintomático en el día, el modelo de manifestación sintomática o microanalítico, pretende dar respuesta a diversas inquietudes que ponen de manifiesto, las diferentes estrategias de medición de la somnolencia excesiva diurna (Pilcher, 2003; Yang, 2004), en cuanto a la operacionalización de lo que se concibe como a manifestación sintomática de las misma (Caskardon 1987; Mitler 1990, 2000).

El análisis comportamental de mani- festación sintomática (Marín, 2005), permite una diferenciación sintomática en la discriminación de las somnolencia, con el objetivo de apuntar mejor a los componentes de sueño diurno y su presencia en el día, como focos centrales en la intervención sintomática de la somnolencia. Plantea que la respuesta de somnolencia excesiva diurna, esta definida como la manifestación de la frecuencia diurna de: 1) Una latencia de sueño inferior a cinco minutos manifestada en, 2) varios episodios sucesivos de sueño diurno entre las 9:00 a.m. y las 6:00 p.m. (se plantea la intermitencia de la misma, en los periodos de las 9:00a.m, 12:30 a.m., 3:00 p.m., y 6:00 p.m.), además, la cantidad de tiempo de sueño diurno (característica que se presenta además del sueño nocturno). Esta frecuencia está delimitada exponencialmente, por la tendencia circadiana, manifestándose en la presencia o no de somnificidad, entendida como la manifestación conductual del sueño diurno, ante situaciones pasivas, o activas (Young, 2004). Estas concepciones se

RSmn $=f(L S+T T S D+C E S D) \times T C \pm S m n f$

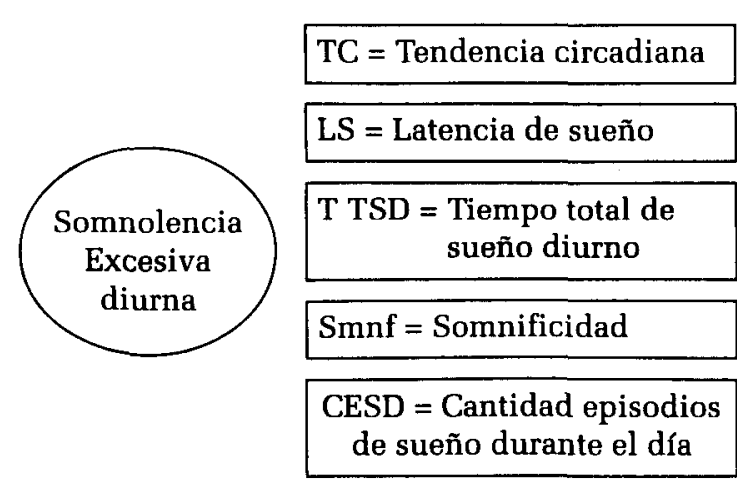

Figura 5. Ecuación del modelo micro analítico de la somnolencia excesiva diurna (Marín, 2005), modificado. La respuesta de somnolencia excesiva (RSmn), es igual a la suma de los factores: latencia de sueño (LS), tiempo total de sueño diurno (TTSD) y cantidad de episodios de sueño durante el día (CESD), por la tendencia circadiana de estos aspectos y más o menos la respuesta de somnificidad (Smnf). 


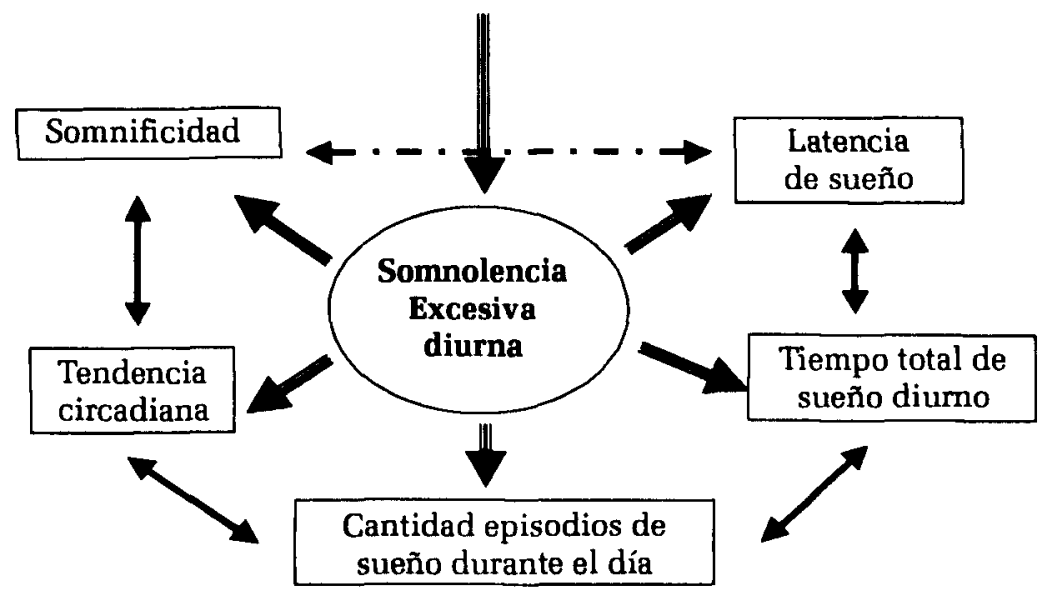

Figura 6. Exposición sintomática de los multicomponente de la somnolencia excesiva diurna, modificado (Marín, 2005). Como se aprecia, se destaca la interacción de los componentes en la manifestación sintomática de la somnolencia, componentes que retoman tanto los modelos de regulación del sueño (en los procesos circadianos y la interacción de los componentes de sueño de la respuesta de la somnolencia), sumados a las diferentes manifestaciones sintomáticas que han descrito varios autores, dentro del test de Latencia Múltiple, la escala de Somnolencia Epworth, la escala de somnolencia de Karolinska, entre otras, y define la interacción de estas respuestas en la manifestación multicomponente de las somnolencia excesiva diurna.

aprecian en las siguientes Figuras 5 y 6 .

\section{EL MODELO COGNITIVO COMPORTAMENTAL}

Después de analizar los diferentes modelos, que explican el sueño y la somnolencia excesiva, se pasa a exponer una explicación comportamental para la narcolepsia, partiendo de dichos conceptos, lo cual en primer lugar permite una comprensión de la sintomatología y el síndrome en sí mismo y en segundo lugar permite una formulación adecuada de los factores tanto intrínsecos, como extrínsecos, detonan los síntomas (Rogers y Mullington, 2003; Marín, 2001b; Hublin et al., 1994; Mamelak, 1992; Okun et al. 2002; Scammell, 2003). Desde las teorías que explican la intervención del síndrome se ha visto necesario las medidas comportamentales además de las físicas, como puntos de partida, para el abordaje del mismo, en conjunción con los procesos farmacológicos, e incluso como medias no tan paliativas para el manejo de los síntomas. Diferentes autores han descrito también y han creado medidas subjetivas de ciertos factores que desencadenan la conducta catapléjica, la cual no es curioso que se manifieste ante situaciones emocionales de arousal alto, como las descritas en la escala de cataplejía de Stanford y su frecuencia en la Escala de Narcolepsia de Ullanlinna (Anic-Labat et al., 1999; Rogers y Mullington, 2003; Hublin et al., 1994; Mamelak, 1992; Okun et al. 2002; Scammell, 2003), estas se pueden observar en la tabla 3, dentro de las cuales se destacan situaciones como risa, enojo, sorpresa, excitación, entre otras. Estas variables hacen pensar en componentes reguladores comportamentales externos y cognitivos internos, como la anticipación a las mismas, similares a los que se presentan en la ansiedad y que sería posible reducir el impacto de las variables físicas, consecuentes del síntoma, utilizando lo mismos mecanismos interventivos formulados en 
Tabla 3. Apartados de las escalas de cataplejía de Stanford (Anic-Labat et al., 1999) y la escala de narcolepsia de Ullanlinna (Hublin et al. 1994). Tanto las conductas del inventario de cataplejía de Stanford como el ítem de cataplejía de la escala de narcolepsia Ullanlinna, hacen pensar en aspectos de regulación tanto intrínsecos como extrínsecos, para la manifestación sintomática del síndrome.

Escala de cataplejía de Stanford

Inventario de episodios ante los cuales se detona el síntoma catapléjico, retomados de la traducción de la escala (Marín).

Cuando ríe

Cuando está enojado

Cuando le excitan

Cuando está sorprendido

Cuando recuerda un momento feliz

Cuando recuerda un acontecimiento emocional

Cuando le requieren hacer una respuesta verbal

rápida en un contexto juguetón o divertido (Ej., un chiste ingenioso)

Cuando le desconciertan

Cuando disciplina a los niños

Durante una relación sexual

Durante actividades atléticas

Después de actividades atléticas

Cuando usted es elogiado

Cuando le tensionan

Cuando usted es el centro de atención

Cuando está tenso

Mientras está jugando un juego emocionante

Cuando tiene un pensamiento o un momento romántico

Cuando usted cuenta u oye una broma

Cuando algo lo mueve emocionalmente
Escala de narcolepsia de Ullanlinna

Situaciones y respuestas de cataplejía evaluadas en el primer ítem de la escala de narcolepsia de Ullanlinna.

¿Cuándo ríe, haciendo algo alegre o enojado, o en una situación emocionante, tiene u ocurren repentinamente los síntomas siguientes?

El doblar de las rodillas

Abertura de la boca

Cabeceo principal

Caerse al piso

Estos items son evaluados con calificaciones de frecuencia, entre: nunca, 1-5 veces durante todo el curso de la vida, mensualmente, semanalmente, diariamente o muchas veces al día diferentes investigaciones sobre en el afrontamiento de conductas ansiógenas severas (Beers, 1979).

El modelo cognitivo-comportamental de la narcolepsia pretende poner en orden, dentro de un modelo de relaciones causales (tanto intrínsecas como extrínsecas), el conjunto informacional de los sistemas implicados en la modulación y anticipación de la producción de la sintomatología del síndrome, lo cual brindará las bases para diseñar un programa estructurado de intervención del mismo. En general sigue tres principios explicativos en la patogénesis del síntoma, basados en la obra comportamental de Hull. En primer lugar la característica central del modelo es que existe una constate interacción entre los factores de riesgo externos entendido aquellos factores asociados a la sintomatología de la enfermedad (como las situaciones que detonan la cataplejía, Ej situaciones emocionales intensas y de arousal alto), y los factores de riesgo interno, que son aquellos considerados como predictores de la narcolepsia (como los sistemas neuroquímicos y fisiológicos, explicados en el modelo de narcolepsia de Stanford). En segundo lugar el modelo, es una interacción organizativa no lineal, pero si regulada por la covariación de procesos implicados en la producción sintomática de la narcolepsia, agrupados en tres dimensiones a saber (modelo molar de la narcolepsia, Anexo 1): En primer lugar aquellos agrupados en la dimensión temporal del síndrome, en donde se describen los factores de desa- 
rrollo etiológico físico(predisponentes), que dan cuenta de aquellos aspectos hereditarios a nivel orgánico, por la maduración evolutiva (como en el caso del inicio del síndrome, que necesita unas características propias físicas y genéticas, en constante interacción para detonarlo, descritas en los criterios diagnósticos) y la regulación neuroanatómica del síndrome; los factores precipitantes que hacen referencia a como unos individuos, bajo ciertas circunstancias, desarrollan la sintomatología del síndrome, mientras otros que tienen las mismas características de desarrollo, no lo hagan, como en el caso de las respuestas catapléjicas frente a ciertos antecedentes emocionales y frente a otros no, dentro de estos aspectos se encuentran también, los sistemas reguladores del sueño, la somnolencia, y las explicaciones comportamentales objetivas del sueño, por último los factores desencadenantes, entendidos como aquellos que anteceden inmediatamente en la aparición del problema, y aunque están relacionados directamente con el síndrome, no constituyen en si mismos un factor causal determinante (como la tendencia a la evaluación negativa, diferencias individuales y factores cognitivos). En segundo lugar la dimensión interactiva, que agrupa variables de entrada, definidas por condiciones ambientales que regulan la respuesta, variables intercurrentes, ligadas cercanamente a la dimensión temporal. Por último, una dimensión resultado de la covariación de todas las anteriores que especifican la respuesta y retroalimentan sistemas de respuestas motivantes, que alimentan los procesos de mantenimiento intrínseco y extrínseco de los síntomas.

Por último un tercer principio, se basa en que cada una de las dimensiones expuestas interactúan en subprocesos al interior de las mismas (explicación molecular del modelo Anexo 2). Dentro de la dimensión temporal, en los factores pre- cipitantes, se encuentra la regulación del sueño, con sus respectivos componentes, explicados en esta misma revisión (proceso S, C y W), lo mismo que el modelo comportamental; en los factores de desarrollo se encuentras, las diferencias individuales, en torno a la raza (R), sexo (S), edad (E) y patrones de personalidad y sociales, los factores cognitivos, como los conceptos erróneos sobre sueño (CES), maximización cognitiva (MaxC), expectativas poco realistas (EPR), atribuciones falsas, el control del sueño, y creencias incorrectas sobre las estrategias que inducen el sueño (CIEIS). Los factores desencadenantes, en donde se encuentran la tendencia a la evaluación negativa, la arquitectura del sueño propia de la narcolepsia (ej. presencia de una latencia de sueño REM muy corta), lo mismo que la regulación circadiana. Estas variables están en asociación con la dimensión interactiva, porque producen las variables de entrada, en donde el organismo tiene en cuenta las repuestas anteriores ante los estímulos que generan somnolencia/cataplejía (en el modelo designado como N), los factores fisiorgánicos, que activan todo el sistema neurotransmisor que genera las repuestas sintomáticas del síndrome (definido como C), la intensidad del estímulo que evoca estas repuestas denimonado (en el modelo S), la fuerza y generalización de la repuesta y El trabajo necesario para dar respuesta al estimulo de entrada (nombrada en el modelo W). en el siguiente punto están las variables intercurrentes, las cuales preparan al organismo para perpetuar o aprender mecanismos motivacionales intrínsecos, que se convertirán posteriormente en factores de riesgo, dentro de estos están la motivación (entendida en el modelo como K), la inhibición reactiva (no enfrentarse al síntoma, designada como Ir), o la inhibición condicionada (IC), que es aquella que es resultado del aprendizaje de estrategias que generan el 
síntoma, casi en un nivel encubierto, es decir la persona, no sabe que dichas actitudes refuerzan e problema; la fuerza del habito previamente condicionado, que describe como el síntoma se había presentado en situaciones anteriores $(\mathrm{H})$; el potencial de reacción ( $\mathrm{Pr}$ ), que define las características intrínsecas y extrínsecas estructuradas en el sujeto que genera la patología. El potencial de reacción generalizado (Prg), que define la respuesta especifica de la sintomatología de la narcolepsia en un individuo; el umbral de reacción (Ur), hace referencia a las condiciones necesarias para emitir una repuesta sintomática de la narcolepsia. Por ultimo las variables de salida en la dimensión resultado de la covariación y respuesta sintomática de la narcolepsia, en donde existen características de las variables de salida asociadas a los síntomas y que pueden producir mecanismos que refuerzan la aparición de los mismos nuevamente, dentro de estas están la latencia de la reacción sintomática (Lrs), la amplitud de dicha reacción (A), y por ultimo el número de respuestas no reforzadas a extinguirse (N).

Cabe anotar que tras la manifestación sintomática de la tétrada narcoléptica (cataplejía; alucinaciones hipnagógicas, somnolencia excesiva y parálisis de sueño, teniendo en cuenta que dicha manifestación no se da completa en el $100 \%$ de los casos de narcolepsia), estas o de uno de sus síntomas, estas repuestas se vuelven cíclicas generando cadenas de mantenimiento patológico.

\section{CONCLUSIONES}

El recorrido general de una conceptuación de la narcolepsia y los contenidos comportamentales y emocionales del desencadenamiento de los síntomas, entre los cuales se describen, situaciones poco estimulantes o poco activas que exacerban los síntomas de la somnolencia excesiva diurna; las deficientes estrategias, cognitivo- comportamentales, para neutralizar los síntomas: las conductas que deprivan el sueño nocturno, que aumentan la frecuencia e intensidad de los episodios de cataplejía; la aparición de comportamientos automáticos, es decir, aquellas en las que el individuo realizará cosas sin ser plenamente consciente de ello; llevan a plantear una clara influencia modular de los factores psicológicos, sobre los factores orgánicos dentro de la explicación del síndrome narcolepsia-cataplejía, objetivo de esta exposición (American Academy of Sleep Medicine 2005; American Psychiatric Association, 2002; Rogers y Mullington, 2003; Marín, 2001b).

La historia general de la explicación del síndrome y las conceptuaciones actuales, han llevado a denotar muchos puntos encontrados en cuanto al planteamiento conceptual-explicativo psicologico del síndrome, argumentando sólo un fenómeno exclusivamente fisiológico; esto se debe en primer lugar a que las explicaciones hasta ahora habían planteado que la narcolepsia es el único trastorno del sueño, en donde los sistemas fisioanatómicos que regulan el sueño, se encuentran alterados, en marcados con una característica genética fuerte, y las investigaciones psicológicas se han centrado, asociando las conductas subyacentes y sintomatológicas del síndrome y el pobre papel de la intervención psicológica, reduciéndose al plano de la prevención del síntoma (desde la higiene del sueño), desde el apoyo social, para abordar las consecuencias psicosociales del síndrome y explicaciones psicoanalíticas interpretando las relaciones del síndrome y el fenómeno de las ensoñaciones, y como consecuencia abandonando cualquier abordaje interventivo de la enfermedad de manera directa (Rogers y Mullington, 2003; Marín, 2001b). 
El propósito central de esta explicación, parte en primer lugar de los aspectos tanto cognitivos como comportamentales y fisiológicos implicados en la manifestación sintomática del síndrome, retomando la características sintomáticas, como medida importante en el planteamiento del modelo, debido a que la narcolepsia hasta ahora es considerada como una enfermedad crónica, por lo tanto cualquier explicación que abogue por una formulación biopsicosocial que permita un acercamiento interventivo, nos remite a una explicación sintomática que propenda por la disminución del impacto de los síntomas abordándolos intrínsecamente como conducta objetivo, en la intervención, debido al mismo peso de la alteración fisioanatómica de la enfermedad (Rogers y Mullington, 2003; Marín, 2001b).

\section{REFERENCIAS}

Achermann, P., y Borbely, A.A. (2003). Mathematical models of sleep regulation. Frontiers in bioscience, 1, 683-93.

Åkerstedt, T.S., y Folkard, S. (1990). An human model of Sleepiness En Sleep '90, Horne (pp. 310-313) Bochum, Germany: Pontenagel press.

Aldrich, M. S. (1991-1992). The neurobiology of narcolepsy-cataplexy syndrome. International Journal of Neurology. 25-26, 29-40.

Aldrich, M.S. (1993). The neurobiology of narcolepsy-cataplexy. Progress Neurobiology, 4, 533-41

Aldrich, M.S., Prokopowicz, G., Ockert, K., Hollingsworth, Z., Penney, J.B. y Albin, R.L. (1994). Neurochemical studies of human narcolepsy: Alpha-adrenergic receptor autoradiography of human narcoleptic brain and brainstem. Sleep, 17, 598-608.

American Academy of Sleep Medicine (2005). International classification of sleep disorders revised: Diagnostic and coding manual. Rochester, Minnesota: Autor.

American Psychiatric Association (2002). Manual diagnóstico y estadístico de los trastornos mentales, DSM-IV-TR (texto revisado). Barcelona: Masson.
Anic-Labat, S., Guilleminault, C., Kraemer, H., Meehan, J., Arrigoni, J., y Mignot, E. (1999). Validation of cataplexy questionnaire in 983 sleep disorders patients. Sleep 22, 77-87

Beers, T., y Karoly, P. (1979). Cognitive Strategies, expectancy and coping style in the control of pain. Journal of Consulting and Clinical Psychology, 47, 179-180

Beersma, D.G. (1998). Models of human sleep regulation. Sleep Medicine Reviews, 2, 31-43.

Billiard, M., Seignalet, J., y Eliaon, J.F. (1994). La somnolence pathologique. La narcolepsie. En M. Billiard (Ed.), Le sommeil normal et pathologique (pp. 261-274). Masson. London

Bladin, P.F. (2000) Narcolepsy-cataplexy and psychoanalytic theory of sleep and dreams. Journal of the history of the neurosciences, 9, 203-17.

Boehme, R.E., Baker, T.L., Mefford, I., Barchas, J., Dement, W.C. y Ciaranello, R. (1984). Narcolepsy: Cholinergic receptor change in animal model. Life Sciences, 34, 1825-1828.

Buela-Casal, G., y Sierra, J.C. (1995). Los trastornos del sueño. En A. Belloch, B. Sandín, y F. Ramos (Eds.), Manual de psicopatología, Vol. 1 (pp. 381-402). Madrid: McGraw-Hill.

Cano, M.C., Miró, E., Espinosa-Fernández, L., y Buela-Casal, G. (2004). Parámetros subjetivos de sueño y estado de ánimo disfórico. Revista de Psicopatología y Psicología Clínica, 9, 35-48

Carlander, B., Eliaou, J.F., y Billiard, M. (1993). Autoimmune hypothesis in narcolepsy. Neurophysiology, 23, 15-22.

Carpizo-Alfayate y Fernández-López, R. (1998) Fisiopatología de la narcolepsia. Vigilia-sueño, 10 (1Sup)/ Septiembre.

Carskadon, M.A. y Dement, W.C. (1987). Daytime sleepiness: quantification of behavioral state. Neuroscience and Biobehavioral Reviews, 11, 307-317.

Conesa-Peraleja, M., y Izquierdo-Vicario (1998). Narcolepsia: Una propuesta de evaluación neuropsicológica. Vigilia-sueño, 10 (1 Sup)/ Septiembre.

Daniels, E., King, M.A., Smith, I.E. y Shneerson, J.M. (2001). Health-related quality of life in narcolepsy. Journal Sleep Research, $10,75-81$. 
De Sarro, G.B., Ascioti, C., Froio, F., Libri, V., y Mistico, G. (1987). Evidence that locus coeruleus is the site where clonidine and drugs acting at alpha-1, and alpha-2 adrenoceptors affect sleep and arousals mechanisms. British Journal of Pharmacology, 90, 675-85.

Dement, W.C., RechTschaffen, A. y Gulevitch, G. (1966). The nature of the narcoleptic sleep attack. Neurology, 16, 1833.

Dement, W.C., y Vaughan, C. (2000). Al acecho del sueño: La narcolepsia y otros trastornos. El animal nocturno y el reloj biológico. En Dormir Bien (pp. 82-108). México: Atlantida.

Dinges, D.F. (1989). The nature of sleepiness: causes, contexts and consequences. En A. Stunkard y A. Baum (Eds.), Perspectives in behavioral medicine: Eating, sleeping, and sex (pp. 147-179). Hillsdale: LEA.

Dinges, D.F. y Kribbs, N.B. (1991). Performing while sleepy: effects of experimentallyinduced sleepiness. En T.H. Monk (Ed.), Sleep, sleepiness, and performance (pp. 97-128). New York: John Wiley \& Sons.

Folkard, S., y Åkerstedt, T.S. (1992). The three-process model of the regulation of alertness-sleepiness. En R.J. Broughton y R.D. Ogilvie RD (Eds.), Sleep, arousal, and performance. A tribute to Bob Wilkinson (pp. 11-26). Boston: Birkhäuser.

Gelineau, J.B.E. (1880). De la narcolepsie. Gazettede Hôpiteau, 53, 626-628.

Gilleminault, C., Henzel, R., Mignot, E. y BlacK, J. (1998). Investigations in to the neurologic basis of narcolepsy. Neurology, 50, S8-S15.

Godbout, R., y Montplaisir, J. (1986). The effect of zimelidine a serotonin reuplake bloker, on cataplexy and daytime sleepness of narcoleptic patients. Clinical Neuropharmacoogyl, 9, 46-51.

Guilleminault, G., y Anagnos, A. (2000). Narcolepsy. En M. Kryeger, T. Roth, y W. Dement (Eds.), Principles and practice of sleep medicine (3rd ed., pp. 676-687). Filadelfia: WB Saunders Company.

Hublin, C., Kaprio, J., Partinen, M., Koskenvuo, M. y Heikkila, K. (1994). The Ullanlinna Narcolepsy Scale: validation of a measure of symptoms in the narcoleptic syndrome. Journal of Sleep Research, 3, 52-59.

Hublin, C., Kaprio, J., Partinen, M., Koskenvuo, M., Heikkila, K., Koskimies, S. y Gui- lleminault, C. (1994) The prevalence of narcolepsy: an epidemiological study of the Finnish Twin Cohort. Annales of Neurology, 35, 709-716.

Hublin, C., Partinen, M., Kaprio, J., Koskenvuo, M. y Guilleminault, C. (1994). Epidemiology of narcolepsy Sleep, 17, S7-12.

Kripke, D. (1976) Biological rhythm disturbances night cause narcolepsy. En C. Guilleminault, W.C. Dement y P. Passouant (Eds.), Narcolepsy (pp. 457-483). New York: Spectrum Publication.

Lock, C.B., So, A.K.L., Welch, K.I., Parkes, J.D., Trowsdale, J. (1998). MHC class II sequences of an HLA-DR2 narcoleptic. Immunogenetics, 27, 449-55.

Loewenfeldt, L. (1902). Ueber narkolepsie. Munchner Medizinische Wochenschrift. 49, 1041-1045.

Mamelak, M. A. (1992). Perspective on narcolepsy. Encephale, 18, 347-51.

Marín, A. (2001a). Factores culturales que deprivan el sueño: Diseño de un inventario.Trabajo investigativo. Manuscrito no publicado, Medellín, Clínica del Sueño Medellín.

Marín, A. (2001b). Estado del arte sobre estudios actuales en hipersomnias. Trabajo investigativo para optar al Título de Psicólogo, Facultad de Psicología, Universidad Cooperativa de Colombia, Colombia.

Marín, H.A. (2005). Calidad de vida y somnolencia excesiva diurna en estudiantes universitarios. Tesis para optar al título de Master en Psicología, Facultad de Psicología, Universidad de San Buenaventura, Medellín, Colombia

Marín, A. y Vinaccia, S. (2004a). Evaluación y tratamiento de la somnolencia excesiva diurna: una revisión. Psicología y Salud, 14, 245-255.

Marín, A., y Vinaccia, S. (2004b). Calidad de vida y somnolencia excesiva diurna. En L. Oblitas (Ed.), Manual de psicología de la salud y hospitalaria. Libro electrónico. www.psicologiacientificaonline.com.

Marín, H.A. y Vinaccia, S. (2005b). Contribuciones desde la medicina comportamental del sueño, al manejo de la somnolencia excesiva diurna. Psicología Desde el Caribe, 15, 95-116.

Marín, H.A., Rodríguez, S., Vivanco, D., Aristizabal, N., Berrio, M., y Vinaccia, S. 
(2005a). Factores culturales que privan el sueño y causan somnolencia excesiva, en estudiantes universitarios: estudio piloto. Psicología y Salud, 15, 57-68.

Mignot, E. (2000) Pathophisiology of Narcolepsy. En M. Kryeger, T. Roth, y W. Dement, (Eds.), Principles and practice of sleep medicine (3rd ed., pp. 663-675). Filadelfia: WB Saunders Company.

Mignot, E. (2001) A commentary on the neurobiology of the hypocretin/orexin system. Neuropsychopharmacology, 25, S5-13.

Mignot, E., Guilleminault, C., Bowersox, S., Frusthofer, B., Nishino, S., Maddaluno, J., Ciaranello, R. y Dement, W.C. (1989). Central a1 adrenoceptor subtypes in narcolepsy-cataplexy: a disorder of REM sleep. Brain Research, 490, 186-91

Mignot, E., Guilleminault, C., Bowersox, S., Rappaport, A. y Dement, W.C. (1988). Effect of alpha 1-adrenoceptors blockade with prazosin in canine narcolepsy. Brain Research, 444, 184-8.

Mignot, E., Guilleminault, C., Bowersox, S., Rappaport, A., y Dement, W.C. (1988) Role of central alfa 1 adrenoceptors in canine narcolepsy. Journal of Clinical Investigation, 82, 885-894.

Mignot, E., Guilleminault, C., Dement, W.C., y Grumet, C. (1992). Genetically determined animal models of narcolepsy, a disorder of REM sleep. En P. Driscoll (Ed.), Genetically-defined animal models of neurobehavioral dysfunction (pp. 90-110). Cambridge, MA: Birkäuser Boston.

Mignot, E., Renaud, A., Nishino, S., Arrigoni, J., Guilleminault, C., y Dement, W.C. (1993). Canine cataplexy is preferentially controlled by adrenergic mechanisms: evidence using monoamine selective uptake inhibitors and release enhancers. Psychopharmacology, 113, 76-82.

Mitler, M.M., Carskadon, M.A., y Hirshkowitz, M. (2000). Evaluating sleepiness. En M. Kryger, T. Roth y W. Dement (Eds.), Principles and practice of sleep medicine (3rd ed., pp. 1251-1257). Philadelphia: W.B. Saunders.

Monk, T.H. (1991). Circadian aspects of subjective sleepiness: A behavioral messenger? En T.H: Monk (Ed.), Sleep, sleepiness, and performance (pp. 39-63). New York: John Wiley \& Sons.
Newman, J. y Broughton, R. (1991) Pupillometric assessment of excessive daytime sleepiness in narcolepsy-cataplexy. Sleep, 14, 121-129.

Okun, M.L., Lin, L., Pelin, Z., Hong, S., y Mignot, E. (2002). Clinical aspects of narcolepsy-cataplexy across ethnic groups. Sleep, 25, 27-35.

Partinen, M., Hublin, C., Kaprio, J., Koskenvuo, M., y Guilleminault, C. (1994). Twin studies in narcolepsy. Sleep, 17, S13-16.

Pilcher, J., Schoeling, J., Scott, E., Prosansky, L., y Caryn, M. (2000, Winter). Self-report sleep habits as predictors of subjective sleepinessStatistical data included. Behavioral Medicine. Encontrado en http://www.findarticles.com.

Pilcher, J.J., Pury, C.L.S., y Muth, E. R. (2003). Assessing daytime sleepiness: An internal state versus behavior approach. Behavioral Medicine, 29, 60-67.

Ramos-Platón, M.J. (1998). Procesos cognitivos y adaptación psicosocial en la narcolepsia. Vigilia-sueño, 10 (1Sup).

Rogers, A.E., y Mullington, J. (2003). The Symptomatic Management of Narcolepsy. En M.L. Perlis y K.L. Lichstein (Eds.), Treating Sleep Disorders: Principles and Practice of Behavioral Sleep Medicine (pp. 118135). New Jersey: Wiley publishers since.

Scammell, T.E. (2003). The neurobiology, diagnosis, and treatment of narcolepsy. Annals Neurology, 53, 154-166.

Stepanski, E. (2002). The effect of sleep fragmentation on daytime function. Sleep, 25, 268-276

Webb, W.B. (1988). An objective behavioral model of sleep. Sleep, 11, 488-496.

Wing, Y.K., Li, R.H., Ho, C.K., Fong, S.Y., Chow, L.Y., y Leung, T. (2000). A validity study of Ullanlinna Narcolepsy Scale in Hong Kong Chinese. Journal of Psychosomatic Research, 49, 355-361.

Wing, Y.K., Li, R.H., Lam, C.W., Ho, C.K., Fong, S.Y., y Leung, T. (2002). The prevalence of narcolepsy among Chinese in Hong Kong. Annals Neurology, 51, 578-584.

Yoss, R.E., y Daly, D.D. (1960). Narcolepsy. The Medicals Clinics of North America, 44, 955-968.

Young, T.B. (2004). Epidemiology of daytime sleepiness: definitions, symptomatology, and prevalence. Journal of Clinical Psychiatry, 65, 12-16. 


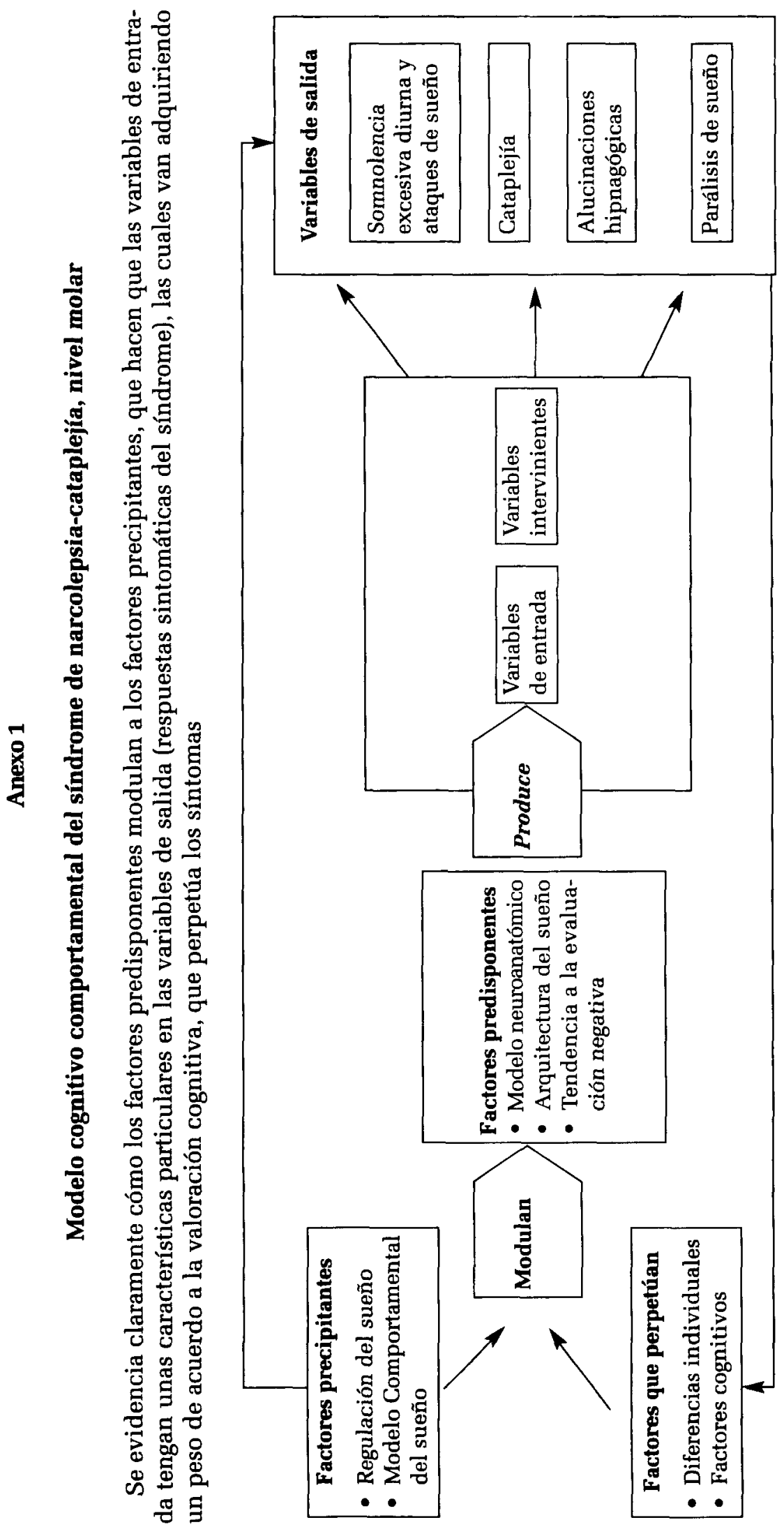


Anexo 2

\section{Modelo cognitivo comportamental del síndrome narcolepsia-cataplejía, nivel molecular}

Se observan ahora las variables antes explicadas que componen cada uno de los factores y la dinámica de los mismos. Factores predisponentes: Regulación del sueño (proceso S, C y W). Sistemas comportamentales, respuesta de sueño (Rs), demanda de sueño (Ds), tendencia circadiana ( $\mathrm{Tc}$ ), factores facilitadores e inhibidores comportamentales del sueño (FICom), además los aspectos del sistema comportamental de la somnolencia excesiva diurna: respuesta de somnolencia excesiva (RSmn), latencia de sueño (LS), tiempo total de sueño diurno (TTSD), cantidad de episodios de sueño durante el día (CESD), tendencia circadiana de estos aspectos y respuesta de somnificidad (Smnf). Factores que perpetúan: diferencias individuales como raza (R), sexo (S), edad (E), factores cognitivos como conceptos erróneos sobre sueño (CES), maximización cognitiva (MaxC), expectativas poco realistas (EPR), creencias incorrectas sobre las estrategias que inducen el sueño (CIEIS), Modulan factores predisponentes, como el modelo neuroanatómico de la narcolepsia: hiperactividad colinérgica (Hiper C), déficit de hipocretina (DH), hipoactividad dopaminergica (Hipo D), aspectos relevantes a la arquitectura del sueño propios de la narcolepsia, y la tendencia a la evaluación negativa del ambiente, tras la manifestación sintomática de la cataplejía. Estos aspectos modulan la producción de variables de entra$\mathrm{da}$, como estímulos que generan somnolencia/cataplejía (N), factores fisiorgánicos, (C), intensidad del estímulo (S), fuerza del habito previamente condicionado $(\mathrm{H})$, fuerza y generalización de la repuesta y trabajo necesario para dar respuesta al estimulo de entrada (W), y las variables intervinientres, mecanismos motivacionales intrínsecos $(K)$, la inhibición reactiva, es decir, no enfrentarse al síntoma, (Ir), inhibición condicionada (IC), el potencial de reacción (Pr), potencial de reacción generalizado ( $\mathrm{Prg}$ ), el umbral de reacción (Ur), que entran en relación con los procesos de las variables de salida y estos a su vez con los síntomas: la latencia de la reacción sintomática (Lrs), la amplitud de reacción (A), número de respuestas no reforzadas a extinguirse (N). 


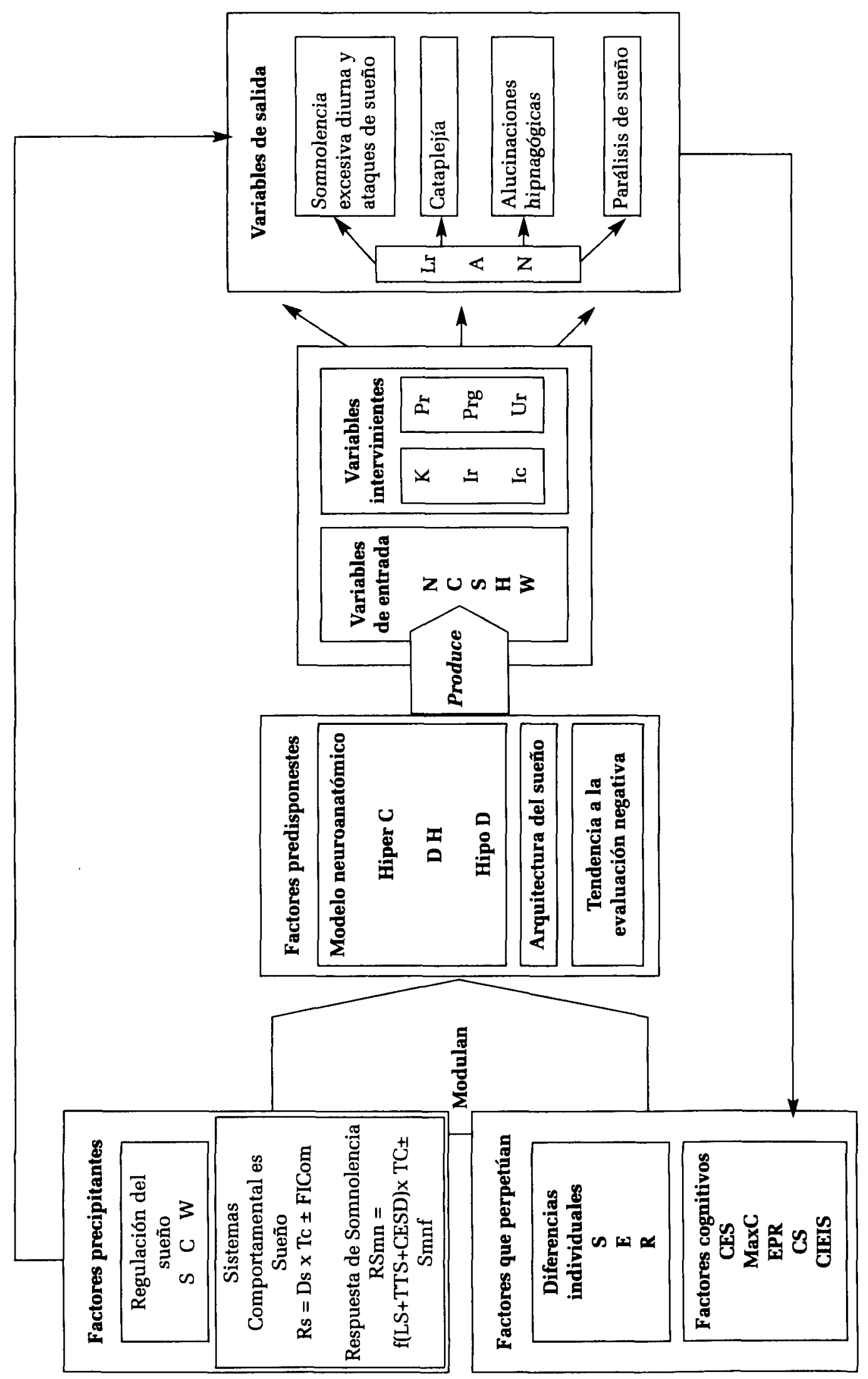

